Vol. 8, Issue 2, February 2019

\title{
Application for Distinguishing Grounds Understudy Troubles Utilize Machine Learning
}

\author{
Mr. Biju Balakrishnan ${ }^{1}$, Adharsh M R ${ }^{2}$, Sharath Prakash ${ }^{3}$ \\ Associate Professor, Computer Science and Engineering, JCT College of Engineering and Technology, \\ Coimbatore, $\operatorname{India}^{1}$ \\ Student, Computer Science and Engineering, JCT College of Engineering and Technology, \\ Coimbatore, India ${ }^{2,3}$
}

\begin{abstract}
The understudy's execution expectation is an imperative research theme since it can enable teachers to keep understudies from dropping out before final tests and distinguish understudies that need extra help. So Accurately foreseeing understudies' future execution dependent on their progressing scholarly records is vital for successfully completing important instructive intercessions to guarantee understudies' on-time and palatable graduation. With the help of machine learning technology, using decision tree algorithm the academic performance and extra-curricular activities of a student will be monitored and learnt to provide alert to the respective tutor. Based on this alert sent by the automated system, the performance of a student could be improved effectively.
\end{abstract}

Keyword: Machine Learning, Decision tree

\section{I.INTRODUCTION}

Despite the fact that there is a rich writing on foreseeing understudy execution when taking care of issues or considering for courses utilizing information driven methodologies, anticipating understudy execution in completing the course is significantly less examined and faces new difficulties: (1) Students contrast immensely regarding foundations and chose courses; (2) Courses are not similarly useful for making precise expectations; (3) Students' advancing advancement should be fused into the forecast. In this paper, we build up a AI and Machine Learning technique for anticipating understudy execution in degree programs that can address these key difficulties. Also, a variety of characteristic language planning strategies were utilized to decide the ideal pre-processing arrangement to create significant outcomes. We found that identifying potential understudy dissatisfaction or disarray was best utilizing a Sequential Minimal Optimization calculation (SMO), and a custom word reference to help decide pertinence likelihood.

\section{EXISTING SYSTEM}

The existing system only detecting potential student frustration or confusion and a custom dictionary to help determine relevance probability. The accuracy of the system is very low and also more complicated. Right now, most of the colleges give utilizing e- learning frameworks regulated learning and, eventually to the precision showed in between rather unwavering quality testing. Going ahead, we hope to proceed with our work on two principle fronts. Initially, to refine the credit recognizable proof to more readily decide course material or points where all understudies normally experience issues, in light of the fact that the probabilities around importance would in general decide in favour of incorporation. Also, second, to test the classifiers created with our present information crosswise over various space and subject materials. At long last, will work grow the utilization of Tutor Alert through the production of modules or coordination into prevalent web-based learning conditions.

\section{PROPOSED SYSTEM}

In proposed framework like those utilized in feeling examination, yet connected to the information created by understudies in a grounds domain so as to recognize confounded or disappointed understudy. To refine the credit distinguishing proof to all the more likely decide course material or points where all understudies commonly experience issues, on the grounds that the probabilities around pertinence would in general decide in favour of incorporation. Also, second, to test the classifiers created with our present information crosswise over various area and subject materials. Will work extend the utilization of TutorAlert through the making of modules or coordination into well-known web-based learning situations. The main advantage of the proposed is there no complication and better to use decision tree algorithm level and also easy and accurate. 
Vol. 8, Issue 2, February 2019

Choice tree constructs arrangement or relapse models as a tree structure. It separates a dataset into littler and littler subsets while in the meantime a related choice tree is steadily created. The last outcome is a tree with choice hubs and leaf hubs. A choice hub has at least two branches. Leaf hub speaks to an arrangement or choice. The highest choice hub in a tree which compares to the best indicator called root hub. Choice trees can deal with both absolute and numerical information.

\section{Decision tree}

\begin{tabular}{|l|l|l|l|l|l|}
\hline \multicolumn{5}{c|}{ Predictors } & Target \\
\hline $\begin{array}{l}\text { Academic } \\
\text { Results }\end{array}$ & $\begin{array}{l}\text { Extracurric } \\
\text { ular } \\
\text { Activities }\end{array}$ & $\begin{array}{l}\text { Surveys / } \\
\text { feedbacks }\end{array}$ & Behaviour & $\begin{array}{l}\text { Final } \\
\text { Result }\end{array}$ \\
\hline low & High & Not satisfy & Poor & Fail \\
\hline Better & No & Satisfy & Good & Pass \\
\hline Good & High & Not satisfy & Very good & Pass \\
\hline
\end{tabular}

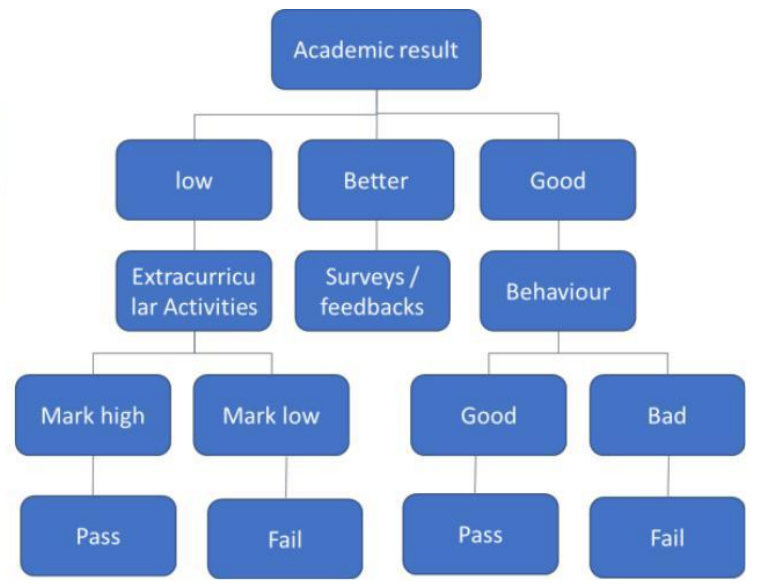

\section{ALGORITHM}

The centre calculation for building decision trees called ID3 by J. R. Quinlan which utilizes a best down, voracious hunt through the space of conceivable branches with no backtracking. ID3 utilizes Entropy and Information Gain to develop a choice tree. In ZeroR model there is no indicator, in OneR model we endeavour to locate the absolute best indicator, credulous Bayesian incorporates all indicators utilizing Bayes' standard and the autonomy presumptions between indicators however choice tree incorporates all indicators with the reliance suppositions between indicators.

\section{Entropy}

A choice tree is constructed top-down from a root hub and includes dividing the information into subsets that contain examples with comparative qualities (homogenous). ID3 calculation utilizes entropy to ascertain the homogeneity of an example. In the event that the example is totally homogeneous the entropy is zero and if the example is a similarly separated it has entropy of one.

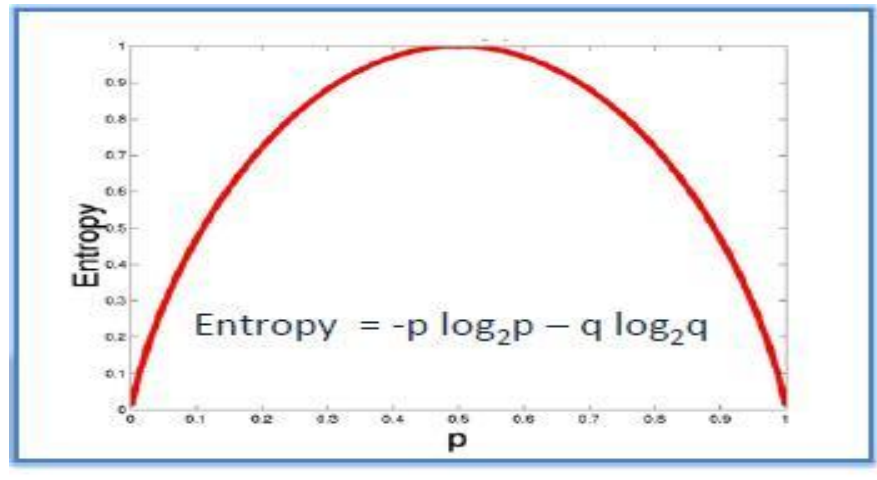

Entropy $=-0.5 \log _{2} 0.5-0.5 \log _{2} 0.5=1$

To build a decision tree, we need to calculate two types of entropy using frequency tables as follows: 1) Entropy using the frequency table of one attribute: ()$=\sum_{=1}^{-} \quad 2 P i$ 2) entropy using the frequency table of two attribute: $(, \quad)=\sum_{\epsilon} \quad \mathrm{P}(\mathrm{c}) \mathrm{E}(\mathrm{c})$. 


\section{ARCHITECTURE}

The overall architecture is given below diagram. The first stage consists of collecting the data like the way of ordinary ERP system and also collecting additional details that includes the individual feedback and surveys etc. these raw data to be maintain in a database then pre-process to classify the data and check relevance and send predicted result of each subject and send to correspond staff

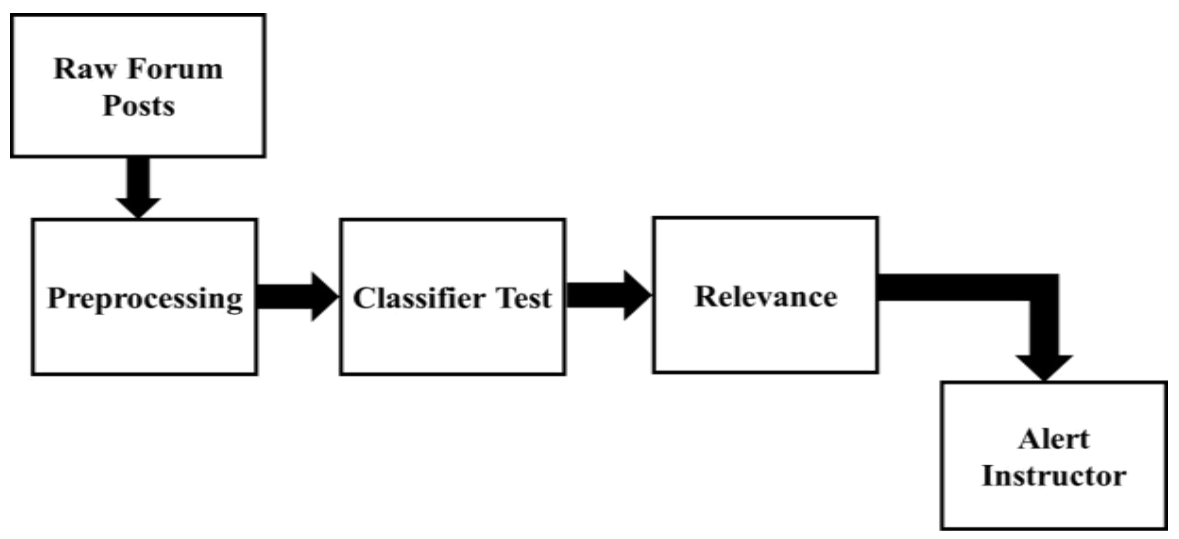

Dataset Student information used to test our strategy is gathered from the Mechanical and Aerospace Engineering division at UCLA crosswise over understudies who graduated in three years $(2013,2014,2015)$. The dataset has 1169 anonymized college understudies joined up with the program in two unique regions (Mechanical Engineering and Aerospace Engineering). All understudies finished their program. We prohibited the exchanged understudies to UCLA since the course data of these understudies before the exchange is absent. The information of every understudy contains the understudy' pre-school qualities (secondary school GPA and SAT scores), the courses (counting addresses and labs) that the understudies take in every scholarly quarter, the course credits and the got evaluations. We utilize just secondary school GPA and SAT scores as the static highlights of understudies because of the restriction of the dataset. In any case, different highlights, if accessible, can be utilized in our structure. UCLA embraces a quarter framework and subsequently, every scholastic term is a quarter. We consider just the fall, winter and spring quarters however not the mid year quarter which has an alternate enlistment design and diverse course terms (for example shorter than courses in different quarters). We consider just letter-grade courses yet not pass/fail courses.

\section{EXPERIMENTAL SETUP}

Ordering exchange posts of an internet learning condition raises some intriguing difficulties. Initially, there are the standard challenges of taking care of freestyle content and the uncertain idea of the English language. Second, there is the need to recognize the subjects of the content, and Endeavor to coordinate them with some learning idea or course highlight to better in from the Instructor of the idea of the trouble. Third the utilization of vocabulary-based arrangements in light of the fact that entangled on the grounds that most broad supposition lexicons or word records that recognize language as positive or negative don't really apply. For example, it is truly conceivable that an understudy could ask for help with a way that was exceptionally positive in language and notion yet may in any case require a similarly expeditious reaction from the Instructor. Various learning strategies were including Support Vector Machines (SVM), Naive Bayes, and Random Forest Algorithm, and a variety of common language pre-preparing strategies were utilized decide the ideal order techniques, and to build the exactness of the outcomes. During preprocessing, stemming is connected to bunch words to their root, a fundamental stop word expulsion is completed to overlook regular words that don't support characterization, and the tokenization is connected. The subsequent information speaks to an unordered accumulation of potential highlights. This element list is additionally refined by weighting the events of potential highlights and utilizing that weighting to help appoint significance probabilities on the difficulty-marked posts.

Another test inalienable to characterizing posts from an advanced classroom talk gathering is the broadness of spaces that an answer should represent. Generally speaking, opinion investigation classifiers and procedures don't exchange promptly from one area to the next, and comparably the language used to distinguish issues with an English course will probably be uniquely not the same as that of a Computer Science course. Any arrangement considering this zone would need to handle this issue utilizing something like Pan et al. 's Spectral Feature Alignment calculation to make a standard connection between the two areas, or use build up the previously mentioned custom word list as a component of an entropy-based classifier as recommended by Deshmukh and Tripathy. For the improvement of TutorAlert, important course material was utilized to physically create the custom. 


\author{
Vol. 8, Issue 2, February 2019
}

\title{
VI CONCLUSION
}

In this paper we have described the understudy of finding student difficulties and predicting the subject results in easy and fastest way. This will also implement in different streams like office, factory for finding the non-working staffs and experience of workers.

Going forward, we discuss this paper to refine mainly two things. First, to refine the credit distinguishing proof to all the more likely decide course material or subjects where all understudies ordinarily experience issues, in light of the fact that the probabilities around significance would in general decide in favour of incorporation. What's more, second, to test the classifiers created with our present information crosswise over various space and subject materials. At long last, will work grow the utilization of TutorAlert through the formation of modules or mix into famous web-based learning conditions.

\section{REFERENCES}

[1]. Singh, V., Saxena, P., Singh, S., \& Rajendran, S. (2017). Opinion Mining and Analysis of Movie Reviews. Indian Journal of Science and Technology, 10(19).

[2]. Elghazaly, T., Mahmoud, A., \& Hefny, H. A. (2016, March). Political sentiment analysis using twitter data. In Proceedings of the International Conference on Internet of things and Cloud Computing (p. 11). ACM.

[3]. Sahayak, V., Shete, V., \& Pathan, A. (2015). Sentiment Analysis on Twitter Data. International Journal of Innovative Research in Advanced Engineering (IJIRAE), 2(1), 178-183.

[4]. Eibe Frank, Mark A. Hall, and Ian H. Witten (2016). The WEKA Workbench. Online Appendix for "Data Mining: Practical Machine Learning Tools and Techniques", Morgan Kaufmann, Fourth Edition, 2016.

[5]. AbuSaaA(2016)Educationaldataminingandstudents'performanceprediction.IntJAdvComputSciAppl. https://doi.org/10.14569/IJACSA.2016.070531

[6]. The White House, "Making college affordable," https:// www.whitehouse.gov/issues/education/higher- education/ making-college-affordable, 2016.

[7]. J. Xu, T. Xing, and M. van der Schaar, "Personalized course sequence recommendations," IEEE Transactions on Signal Processing, vol. 64, no. 20, pp. 5340-5352, Oct 2016. 